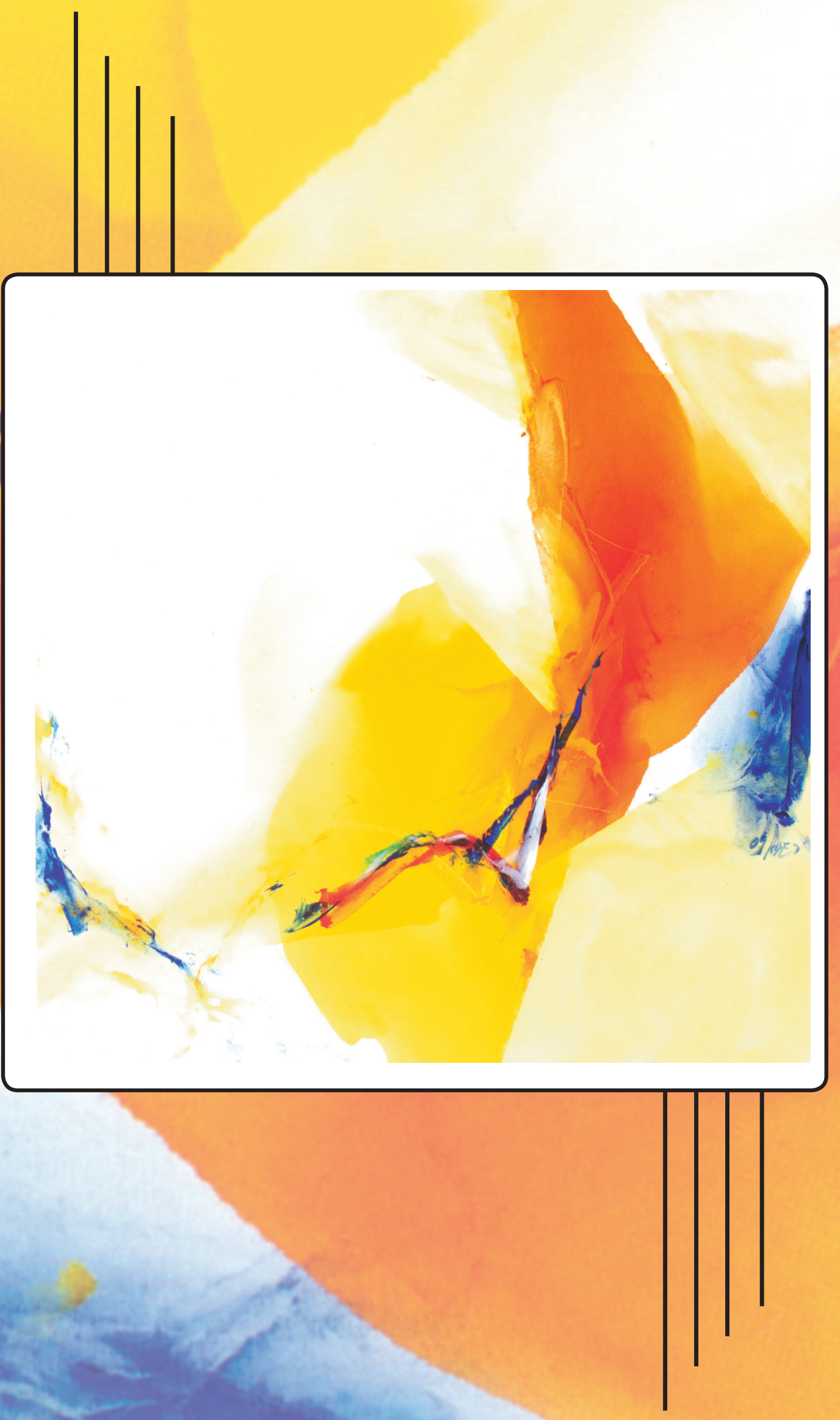


Hommage aux 12 apotres

Óleo sobre Lienzo

$120 \times 120 \mathrm{cms}$

2009

Colección Privada Universidad Santo Tomás 


\title{
DIAGNÓSTICO DEL DESEMPEÑO DE LOS EGRESADOS TOMASINOS DE LA FACULTAD DE CONTADURÍA PÚBLICA
}

\author{
Luis Eusebio Valdivieso Rodríguez ${ }^{l}$ \\ Elizabeth Lozano Vera ${ }^{2}$
}

\section{RESUMEN}

El objetivo general del proyecto es el de evaluar el impacto que tiene en la región el programa de Contaduría Pública de la Universidad Santo Tomás Seccional Bucaramanga a partir de las expectativas y necesidades de los empresarios, las aspiraciones de la sociedad y el campo de acción de los egresados, con el propósito de adoptar políticas que garanticen el reconocimiento del programa en el contexto y en sus procesos de mejoramiento y calidad académica de sus estudiantes.

En su ejecución se aplicaron instrumentos a egresados y empresarios cuya información luego de procesada y evaluada nos permitió obtener conclusiones que favorecieron la formulación de juicios sobre la incidencia que el programa ha tenido en la comunidad.

\section{PALABRAS CLAVE}

Egresados tomasinos, Contador público, Contadores tomasinos.

\begin{abstract}
The broad objective of the project is to evaluate the impact the Public Accounting Program of the Universidad Santo Tomas, Bucaramanga Section has had on the region, from the expectations and needs of entrepreneurs, the aspirations of the society and the domain of action of the Alumni with the goal to adopt policies to guarantee the recognition of the program in the process of enhancement of itself as well as the academic quality of its students.

In the project, both Alumni and entrepreneurs were surveyed to obtain valuable feedback and information that was processed and evaluated to formulate important conclusions on the impact of the program on the Community.
\end{abstract}

\section{KEY WORDS}

Public Account, Santo Tomás Alumni, Contador público, Santo Tomas Public Account.

\footnotetext{
1 Especialista en Auditoria medioambiental, Decano y Docente Investigador Universidad Santo Tomás

2 Especialización: Actualización y perfeccionamiento de métodos y técnicas avanzadas de investigación, aplicadas a la educación. Docente-Investigador Universidad Santo Tomás
} 


\section{INTRODUCCIÓN}

Para las entidades que comercializan o fabrican productos así como para aquellas dedicadas a la prestación de servicios, un factor fundamental para el desarrollo y la planeación de sus políticas de crecimiento, expansión, inversión y proyección de sus actividades, es el conocer el grado de aceptación y reconocimiento de los productos que ofrece y servicios que presta, y es acá donde el Contador Público se involucra como profesional frente a las exigencias del desarrollo empresarial.

Para tal fin, la Facultad de Contaduría Pública de la Universidad Santo Tomás, ve la necesidad de formar a sus egresados dentro de un programa que articule la academia con el sector productivo. Observa que frente al mundo globalizado en el que se desarrollan las actividades actualmente, las .disposiciones vigentes en materia de calidad de la educación, que a todos los niveles ha promovido el Gobierno Nacional, el reconocimiento de la calidad académica otorgado a la universidad por el Ministerio de Educación Nacional con el premio Luis López de Meza por la acreditación de algunos de sus programas académicos, entre ellos el de la Facultad de Contaduría Pública, se hace necesario establecer con claridad las perspectivas y proyecciones del programa, conocer los aspectos fundamentales que la han posicionado en la región, establecer políticas en relación con sus egresados, identificar los aspectos por mejorar que le garanticen la permanencia y crecimiento, mantener una comunicación fluida con sus egresados para la realización de programas de extensión a nivel de cursos de actualización, diplomados y programas de posgrado como especialización, maestría y doctorado, que renueven el sentido de pertinencia de los egresados mediante servicios de apoyo integral, que contribuyan a su crecimiento personal y profesional y sirvan de fundamento para la adopción de reformas curriculares.

El reconocimiento en Colombia de la Contaduría Pública como profesión y las reglamentaciones que se dieron a partir de 1.956 se convirtieron en el punto de partida para su extensión a todas las regiones de Colombia donde el desarrollo de los negocios exigía la presencia de personal calificado como su soporte y como una forma de satisfacer las necesidades de carácter legal establecidas por la ley, al exigir la calidad del contador público en su desempeño como Revisor Fiscal en las sociedades que por virtud de la disposición legal estaban obligadas a contar con esta figura. De igual forma el otorgamiento que establece el Art. 10 de la Ley 43 de 1.990 de dar fe pública con la atestación o firma de los actos propios de la profesión, con fundamentos legales que respaldan el ejercicio profesional del Contador Público.

Es de resaltar que la región de Santander cuenta con una importante tradición comercial, a nivel nacional e internacional; es importante destacar la vocación industrial venida a menos en las últimas décadas, en Santander nació la industria textil del país que luego migró a la región antioqueña, en ella tienen asiento industrias importantes del sector de las confecciones y la marroquinería, empresas prestadoras 
de servicios, entidades del sector agropecuario e instituciones del sector solidario que se enmarcan en lo que comúnmente se conoce como pequeñas y medianas empresas, Pymes, un importante porcentaje de ellas es de carácter familiar.

Consecuente con esta situación y requerimientos del entorno, nace como producto de la decisión, el empuje y el entusiasmo de un dominico santandereano Fray Luis J. Torres Gómez, O.P., la Universidad Santo Tomás, Seccional Bucaramanga con las Facultades de Derecho, Economía y Contaduría Pública como respuesta a la necesidad sentida en la sociedad santandereana y de manera muy especial de Bucaramanga y sus alrededores, que junto con Girón, Floridablanca y Piedecuesta conforman el área metropolitana.

La Universidad Santo Tomás seccional de Bucaramanga, fundada el 21 de diciembre de 1972, inició actividades académicas el siete de marzo de mil novecientos setenta y tres. Desde entonces ha ofrecido servicios educativos que siempre han contado con el respaldo de la comunidad en general. Como institución formadora, es consciente del proceso de revisión y renovación que debe mantener para conservar los principios de calidad y mejorar en aquellos aspectos que se considere pertinente hacerlo. Una forma de identificar fortalezas y debilidades es consultar a sus egresados sobre su parecer cuando se enfrentan al ejercicio profesional y a los empresarios que son quienes los ven actuar y resolver las diferentes situaciones que ofrece el desempeño laboral en el medio.

De la Facultad de Contaduría Pública han egresado más de 2.400 profesionales ubicados en diferentes empresas de la ciudad, la región, el país y aún en el exterior. El Programa busca que sus egresados respondan a las expectativas de sus clientes, para ello considera necesario desarrollar competencias entendidas, según Sergio Tobón, como:

\footnotetext{
"Procesos complejos mediante los cuales las personas realizan actividades y resuelven problemas de contexto profesional, mediante la articulación del saber conocer con el saber hacer y el saber ser, con autonomía, conciencia crítica y creatividad, buscando el logro de las metas organizacionales, el desarrollo social y la propia autorización personal" (Tobón, 2003).
}

Estas consideraciones llevaron al grupo a plantearse la siguiente pregunta: ¿Cuál ha sido el impacto que han tenido los contadores públicos egresados de la Universidad Santo Tomás, Seccional Bucaramanga desde su perspectiva y la del sector productivo?

Para indagar por una respuesta a esta inquietud, se propuso como objetivo general evaluar el impacto en la región del programa de Contaduría Pública de la Universidad Santo Tomás, seccional Bucaramanga a partir de las expectativas y necesidades de los empresarios y el campo de acción de los egresados, con el propósito de adoptar políticas que garanticen la satisfacción de la comunidad educativa de la facultad y de la sociedad. 
La identificación de aspectos por mejorar y la adopción de medidas encaminadas a superarlas permitirán junto con los aspectos positivos lograr una mayor fortaleza para enfrentar las amenazas identificadas y las que surjan en continuo trascurrir de la actividad académica. El reconocimiento de puntos débiles o negativos debe reflejarse en una propuesta de mejoramiento sobre aspectos académicos, tecnológicos o metodológicos que permitan a los egresados, empresarios y comunidad en general encontrar en el programa una respuesta permanente de formación vigente que atienda sus expectativas y satisfaga sus necesidades.

\section{Características del ambiente en el que se desenvuelve el futuro profesional de contaduría pública}

Para el desarrollo del trabajo, se tuvo en cuenta partir de algunas características del ambiente en el que se desenvuelve el egresado de la Facultad de Contaduría Pública de la Universidad Santo Tomás, seccional Bucaramanga, se consideró que el egresado es, ante todo, un profesional que sirve como garantía de un adecuado espíritu de servicio y responsabilidad social; se le advierte como un profesional de alto nivel académico y de gran proyección en su servicio y fundamentalmente con un comportamiento ético, uno de los requerimientos más importantes y de mayor exigencia para los contadores públicos

El programa, considera dentro de su política de acompañamiento, en lo que corresponde al fortalecimiento del desarrollo de la personalidad del profesional contable, orientar sus estrategias pedagógicas hacia dos aspectos fundamentales: la técnica del trabajo y la calidad humana. La Universidad Santo Tomás y de hecho la Facultad de Contaduría Pública sustentan la formación de sus egresados en un proyecto de hombre y de sociedad, en un marco adecuado de valores y de conciencia cívico-política que esté a la altura de la crisis y de los retos del nuevo milenio a nivel colombiano, latinoamericano y mundial.

En la Facultad de Contaduría Pública se exige una preparación académica que comprenda todo el fundamento técnico que requiere el profesional para su desempeño exitoso acompañado de un comportamiento moral sin el cual no tendría razón de ser la formación integral que fundamenta la misión institucional. Esto hace imperioso establecer las competencias necesarias para que el contador público, egresado de la facultad, pueda ejercer su actividad profesional con calidad, ajustada a las necesidades y exigencias del sector productivo y satisfaga estándares regionales, nacionales e internacionales. Para lograrlo se busca que los egresados excedan las expectativas de sus clientes y del público, por ello ve la necesidad de aceptar el continuo desafío que involucra la preparación de sus estudiantes orientados hacia la fuerza del conocimiento y de las necesidades de la misma empresa para, de esta forma, establecer la realidad del perfil del contador público que se debe formar en beneficio del contexto. 


\section{Ambientes a los cuales se enfrenta el Contador Público}

Existen nuevos ambientes a los cuales se ve abocado el profesional de Contaduría Pública, que le permita acrecentar día a día su nivel de competitividad con calidad y eficiencia.

Globalización del conocimiento: Significa que el conocimiento producido en un lugar puede ser generalizable y difundido en cualquier otro lugar, independiente de barreras geográficas y culturales. El Contador Público debe sentirse parte de un conglomerado interactivo, que lo debe llevar a una reformulación del lugar que ocupa en su espacio regional, nacional e internacional.

Pluralismo y la diversidad: Cada parte del mundo globalizante desea mantener su identidad y especificidad, en este sentido el reto del Contador Público, egresado de la Universidad Santo Tomás, es garantizar con su formación humanista, el respeto a la divergencia, sin renunciar a sus principios y creencias.

Complementariedad: El egresado debe mostrar lo que mejor hace con respecto a profesionales de otras instituciones e intercambiar con ellos lo que le hace falta. Lo anterior motiva al programa y a la Universidad a procurar en todo momento mejorar los reconocidos niveles de calidad.

Informalidad y permeabilidad: Las instituciones tienden, cada vez, a romper estructuras formales rígidas para flexibilizar su acción y facilitar el acceso a sus recursos por usuarios muy diversos y con objetivos disímiles. Lo anterior implica que los Contadores Públicos rompan con muchas formalidades de acceso a los diferentes espacios de acción, se hagan más abiertos y capaces de prestar servicios, en cualquier momento de su actividad profesional.

Cambio rápido y transitoriedad: El cambio parece ser el denominador común de la sociedad globalizante, que obliga a definir estructuras dinámicas con ciclos de vida menores que el pasado, capaces de adaptarse a los cambios rápidos que garanticen de esta forma la supervivencia. Para el contador público la actualización permanente, la renovación, la capacitación tecnológica, la actitud receptiva y abierta se debe convertir en conocimiento que transforma su quehacer en función de necesidades sociales y empresariales.

\section{MATERIAL Y METODOLOGÍA}

El trabajo investigativo que se desarrolló fue de carácter descriptivo, estructurado, en cuanto que se partió de la recolección de la información a partir de la construcción y aplicación de instrumentos, encuestas, aplicadas a egresados y sector empresarial, se procuró obtener de la fuente principal la información que permitiera evaluar el 
impacto que han tenido los egresados de la Facultad de Contaduría Pública de la Universidad Santo Tomás, seccional Bucaramanga en su desarrollo y desempeño profesional. Para alcanzar los aspectos comentados el proceso se desarrolló en tres etapas o fases.

\section{FASE 1: INFORMACIÓN}

En esta parte el grupo se documentó con los procesos académicos, contenidos, y otros parámetros propios de la carrera que son aportados por la Universidad. Esta información es, a criterio del grupo, piedra angular para determinar procesos valorativos que contribuirán a la generación de la propuesta objeto de este proyecto de investigación.

\section{FASE 2: IDENTIFICACIÓN DEL GRUPO PILOTO}

Se realizó una encuesta piloto a una muestra de la población según base de datos obtenida de los archivos de la Facultad con la finalidad de evaluar puntos de referencia representativos que permitieran realizar los reajustes correspondientes a un trabajo de campo que se acercara más a la realidad del egresado del programa. La encuesta proporcionó parámetros que permitieron determinar la competitividad del egresado tomasino, el cumplimiento de su proyecto de vida como profesional de la contaduría pública; de igual forma el poder establecer si el sector productivo (empresas) genera un discernimiento claro sobre el servicio profesional recibido en esta área del saber. Se desarrolló en tres (3) etapas:

\section{Recolección de la información}

Fue un trabajo de campo, sustentado en la encuesta aplicada a un grupo definido (prueba-piloto) de 24 egresados de la Facultad y que correspondieron al 1\% de una población de 2.423 egresados. Para la aplicación de la encuesta se tuvo en cuenta tres (3) aspectos que se consideraron estrechamente relacionados entre sí: Selección del instrumento de medición, aplicación del instrumento y preparación de las mediciones (Codificación de los datos)

\section{Tabulación de resultados}

La Información se tabuló en cuadros, tablas estadísticas y gráficas circulares de fácil interpretación. En cuanto a la lectura de los mismos la realizaron especialistas y no especialistas. Según la frecuencia de las repuestas obtenidas en cada una de las encuestas, se procedió a tabular y graficar la información con el fin de identificar las consistencias e inconsistencias, que sirvieran de referencia para la elaboración del instrumento definitivo, con el cual se obtuvo al información final. 


\section{Construcción de encuestas a egresados y empresarios}

Se realizaron los reajustes necesarios y se procedió a la elaboración del instrumento definitivo y se aplicó a una muestra representativa de 181 egresados de la Facultad que correspondió al $7.47 \%$ de la población total de 2.423 egresados.

\section{FASE 3: IDENTIFICACIÓN Y APLICACIÓN DE LA ENCUESTA A LA MUESTRA POBLACIONAL}

Se aplicó la encuesta, se tabularon los resultados y se registraron en cuadros y gráficas. Una vez registrados y analizados los resultados se procedió a estructurar y presentar una propuesta a la Facultad que implicara un aporte al proceso de autorregulación. Para la tabulación y análisis de los resultados, se tuvo en cuenta tanto las respuestas de los egresados que laboran en el sector público como en el privado. La aplicación de la encuesta se hizo en forma directa con el encuestado y por correo electrónico. Se resalta la colaboración de los estudiantes de la Facultad para la captación de la información pues contribuyeron como encuestadores con los profesionales egresados del programa y vinculados en las empresas donde ellos realizan su actividad laboral.

La encuesta aplicada tiene validez y confiabilidad en cuanto a que: No fue producto de una improvisación. Hubo conocimiento del proceso que se daría en cuanto a la construcción de la encuesta. El instrumento fue elaborado por el propio grupo de investigadores y ajustado al contexto laboral e institucional con fundamento en su sentido humanista y científico; la encuesta es apropiada para el grupo de egresados, en cuanto se construyó con un lenguaje adecuado a su preparación y comprensión. En cuanto a las condiciones de aplicación, se buscó hacerlo en un ambiente que favoreciera una actitud positiva para resolver la encuesta. En este aspecto se tuvo alguna dificultad expresada por lo extenso del cuestionario. Sin embargo el porcentaje de la población que lo expresó no fue muy significativo.

La encuesta realmente midió lo que se pretendía medir y refleja dominio en aspectos relacionados con:

- Tiempo de ejercicio de la profesión

- Trabajo en actividades relacionadas con la ciencia contable y áreas específicas

- Posicionamiento del contador público en el mercado laboral

- Actitud del egresado frente a la carrera y frente al programa

- Estudios posteriores a su título de contador

- Enfoques de la carrera apropiados para su posicionamiento en el contexto

- El contador público frente a otros campos diferentes del área contable 
- Actividades que podría programar la facultad de carácter académico, cultural y social en espacios diferentes al aula de clase.

\section{RESULTADOS}

Del instrumento aplicado tanto a egresados como al sector productivo, se presentan los datos más significativos y que se considera son fortaleza para establecer el impacto que han tenido los egresados de la facultad de Contaduría Pública en el contexto y su proyección en los procesos de mejoramiento del programa.

Es importante destacar que se contó con la participación de estudiantes y egresados de todas las promociones de la Facultad desde su apertura. La muestra representa todas las generaciones de egresados desde la más reciente hasta aquellos con más de 15 años de culminación de sus estudios, el $80 \%$ de los encuestados tienen cuatro o más años de ejercicio profesional.

Resultaría incompleto el estudio de la evaluación del impacto de los egresados de la Facultad de Contaduría Pública de la Universidad Santo Tomás, seccional Bucaramanga si no se contara con la opinión de los empresarios, su punto de vista es fundamental para reunir los elementos de juicio necesarios, si se desea obtener conclusiones objetivas y concretas, sobre el trabajo realizado. Se encuestaron 30 empresarios.

La región se caracteriza por el predominio de empresas del sector comercial y de servicios que dentro del rango de activos e ingresos son catalogadas en su mayoría como Pymes que son la principal fuente de empleo en la región, de igual forma existe un buen número de ellas de carácter familiar. Para el estudio se consultó la opinión de 30 empresarios y/o representantes legales sobre aspectos de carácter general relacionados con los profesionales de la Contaduría Pública egresados de la universidad Santo Tomás.

Los puntos de referencia para la presentación de los resultados son:

- Relación de la actividad laboral del egresado con el desempeño de actividades propias de la ciencia contable. 
Figura 1. Relación de la actividad laboral del egresado

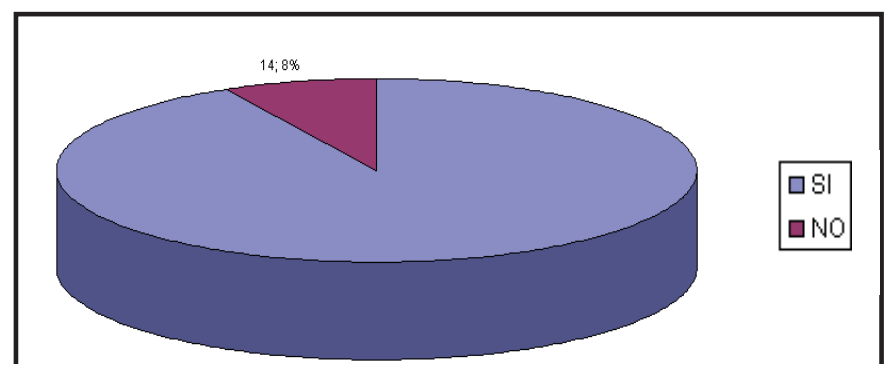

Fuente: Grupo investigador

El 92\% de los egresados encuestados se desempeña laboralmente en actividades propias de la profesión, este indicador refleja el grado de pertenencia del programa y cómo satisface una necesidad de la comunidad que encuentra en estos profesionales el apoyo y acompañamiento para el desarrollo de sus actividades empresariales, el cumplimiento de obligaciones legales y disponer de información confiable para la toma de decisiones.

- Forma como ha desarrollado el contador público su actividad profesional

Figura 2. Forma como desarrolla la actividad laboral el egresado

\section{$167 ; 92 \%$}

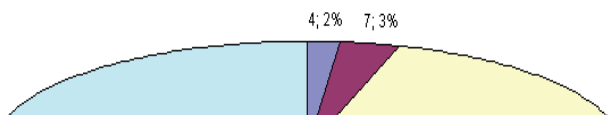

Fuente: Grupo investigador 
El contador público tiene la oportunidad de desempeñarse como empleado, como independiente y como empresario, en desarrollo de su actividad profesional se encuentran en estos campos de manera exclusiva o de manera simultánea dado que mientras se encuentra vinculado laboralmente con una empresa puede realizar actividades de asesoría con otras entidades.

- Sectores de desarrollo de la actividad profesional de los egresados

Figura 3. Sectores de desarrollo de la actividad

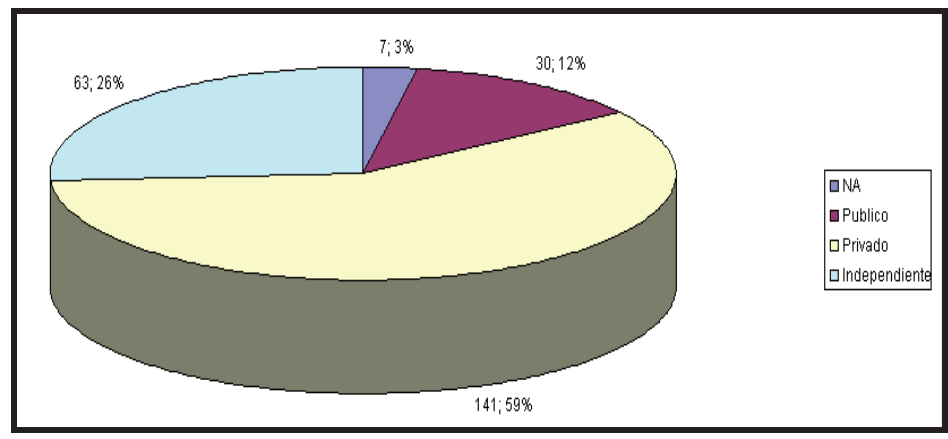

Fuente: Grupo investigador

Si bien es cierto que el sector privado representa el mayor campo de acción de los egresados, el sector público es un campo inexplorado en muchos aspectos y en donde el contador público tiene una excelente oportunidad de participación y proyección.

- Aspectos que se considera se deben tener en cuenta o reforzar en el programa de contaduría pública en cuanto a su formación profesional

Tabla 1. Aspectos que se deben reforzar en el Programa de Contaduría Pública, propuestos por el egresado

\begin{tabular}{lcclcc}
\hline Más práctica & 45 & $19 \%$ & Más charlas y talleres & 2 & $1 \%$ \\
\hline Tecnología & 20 & $9 \%$ & Alta gerencia & 2 & $1 \%$ \\
\hline Tributaria & 19 & $8 \%$ & Revisoría & 2 & $1 \%$ \\
\hline Profundizar en finanzas & 14 & $6 \%$ & Profundizar en presupuesto & 2 & $1 \%$ \\
\hline
\end{tabular}


Diagnóstico del desempeño de los egresados tomasinos...

\begin{tabular}{|c|c|c|c|c|c|}
\hline Ética Profesional & 13 & $6 \%$ & Derecho laboral & 2 & $1 \%$ \\
\hline Normas Internacionales & 11 & $5 \%$ & Bolsa de empleo/Banco de H.V & 2 & $1 \%$ \\
\hline Profundizar en administración & 11 & $5 \%$ & Mayor variedad en diplomados & 2 & $1 \%$ \\
\hline Creación de empresas & 10 & $4 \%$ & Liderazgo & 2 & $1 \%$ \\
\hline Dominio de inglés & 9 & $4 \%$ & Profundizar en Nómina & 1 & \\
\hline Actualizar Normatividad Fiscal & 8 & $3 \%$ & Humanidades & 1 & \\
\hline Profundizar en Costos & 8 & $3 \%$ & Elaboración de declaraciones & 1 & \\
\hline Actualizaciones en auditoría & 8 & $3 \%$ & Comercio exterior & 1 & \\
\hline Análisis de estados financieros & 7 & $3 \%$ & Normas Importación y Exportación & 1 & \\
\hline Investigación & 7 & $3 \%$ & programas en otras ciudades & 1 & \\
\hline Economía de las actualizaciones & 6 & $2 \%$ & Conferencias de desempeño profesional & 1 & \\
\hline Mejorar calidad docente & 6 & $2 \%$ & Mayor continuidad en las materias & 1 & \\
\hline Facilitar contacto con egresados & 5 & $2 \%$ & Plan de mejoramiento continuo & 1 & \\
\hline Profundizar en contabilidad & 3 & $1 \%$ & Contabilidad gerencial & 1 & \\
\hline Formación en mercadeo & 2 & $1 \%$ & Total & 238 & $100 \%$ \\
\hline
\end{tabular}

Fuente: Grupo Investigador

Tabla 2. Aspectos que se deben reforzar en el Programa de Contaduría Pública, propuestos por el sector productivo

\begin{tabular}{l|c|c}
\hline Conocimiento Tecnológico & 14 & $32 \%$ \\
\hline Expresión oral y escrita & 7 & $17 \%$ \\
\hline Práctica empresarial & 3 & $7 \%$ \\
\hline Aspectos gerenciales & 3 & $7 \%$ \\
\hline Aspectos administrativos & 3 & $3 \%$ \\
\hline Planeación tributaria y financiera & 2 & $4 \%$ \\
\hline Dominio de otros idiomas & 2 & $4 \%$ \\
\hline Tratados internacionales & 2 & $4 \%$ \\
\hline Relaciones Públicas & 2 & $4 \%$ \\
\hline Revisoría fiscal & 1 & $2 \%$ \\
\hline Presentación personal & 1 & $2 \%$ \\
\hline Perfil de asesores & 1 & $2 \%$ \\
\hline Mercadeo & 1 & $2 \%$ \\
\hline Innovación & 1 & $2 \%$ \\
\hline Enfoque hacia la generación de empresa & 1 & $2 \%$ \\
\hline Control interno & 1 & $2 \%$ \\
\hline
\end{tabular}

Fuente: Grupo Investigador 
Se considera por parte de los egresados la conveniencia de incrementar y fortalecer las prácticas y la formación tecnológica aspecto en el cual coinciden con los empresarios quienes destacan este punto como el de mayor relevancia, de igual forma destaca el sector productivo la importancia de la comunicación verbal y escrita. Estos puntos de vista son un importante aporte que retroalimenta el proceso académico y son fundamento para la revisión permanente que debe darse sobre el plan de estudios.

- Actividad social, tipo de sociedad y sector al que representan los empresarios

Los empresarios encuestados representan empresas que desarrollan su actividad en el campo industrial en un $13 \%$, comercial en un 50\% y de servicios en un $33 \%$, de las cuales el $17 \%$ pertenecen al sector público y el $83 \%$ al sector privado. Respecto del tipo de sociedad el 50\% corresponde a empresas de responsabilidad limitada, el $23 \%$ a Sociedad anónimas y el $27 \%$ restante a entidades del sector cooperativo y fundaciones, entre otras.

\section{Número, tipo de vinculación y actividad que desarrolla el egresado en el sector productivo}

De acuerdo con la información procesada el 64\% de las empresas cuenta con la colaboración de un egresado, en tanto que en el 36\% restante, el aporte es de más de un egresado. En relación con el tipo de vinculación el 36\% lo hacen como empleados, el $2 \%$ como asesores, como contadores independientes el $33 \%$ y como revisor fiscal el $29 \%$, en relación con la actividad que desarrolla el $76 \%$ se desempeña en actividades propias del ejercicio profesional, el 18\% en actividades de carácter administrativo y el 6\% restante en otras actividades. El desempeño de los egresados está orientado, de forma preferencial al ejercicio de la profesión, sin desconocer que algunas personas puedan participar en la administración y dirección de las empresas, como se desprende de los datos anteriores.

- Apreciación del desempeño de los egresados de la Facultad por el sector productivo 
Figura 4. Calificación del desempeño de los egresados por el sector productivo

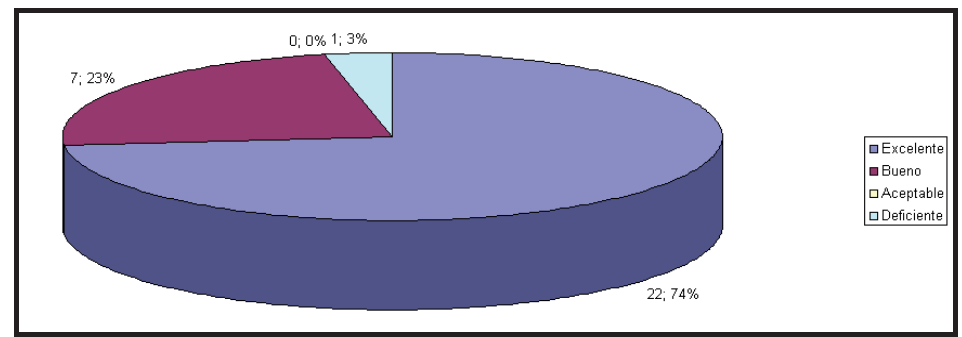

Fuente: Grupo investigador

Tabla 3. Aspectos que identifican un comportamiento como excelente del ejercicio del egresado por el sector productivo

\begin{tabular}{|c|c|c|}
\hline \multicolumn{3}{|c|}{ Razones excelente } \\
\hline Responsabilidad & 14 & $43 \%$ \\
\hline Grandes conocimientos & 8 & $24 \%$ \\
\hline Seriedad & 5 & $15 \%$ \\
\hline Gran experiencia & 2 & $6 \%$ \\
\hline Cordialidad & 1 & $3 \%$ \\
\hline Calidad humana & 1 & $3 \%$ \\
\hline Honradez & 1 & $3 \%$ \\
\hline Permanente actualización & 1 & $3 \%$ \\
\hline Total & 33 & $100 \%$ \\
\hline
\end{tabular}

Fuente: grupo investigador

Tabla 4. Aspectos que identifican un comportamiento como

Bueno del ejercicio del egresado por el sector productivo

\begin{tabular}{lcc}
\hline \multicolumn{3}{c}{ Razones Bueno } \\
\hline Calidad conocimientos & 1 & $14 \%$ \\
\hline Calidad profesional & 1 & $14 \%$ \\
\hline Especialización permanente & 2 & $28 \%$ \\
\hline Conocimientos en tributaria & 1 & $14 \%$ \\
\hline Conocimientos en financiera & 1 & $14 \%$ \\
\hline Capacidades gerenciales & 1 & $14 \%$ \\
\hline Total & 7 & $\mathbf{1 0 0 \%}$ \\
\hline
\end{tabular}

Fuente: grupo investigador 
El concepto de los empresarios refleja un absoluto grado de satisfacción con el desempeño profesional de los egresados y por diferentes razones como la responsabilidad, los conocimientos, la honestidad y su disposición al trabajo como elementos para calificar de manera excelente o buena la actividad de los egresados.

- Aspectos que destacan los empresarios de los egresados del programa

Figura 5. Aspectos que destacan los empresarios de los egresados

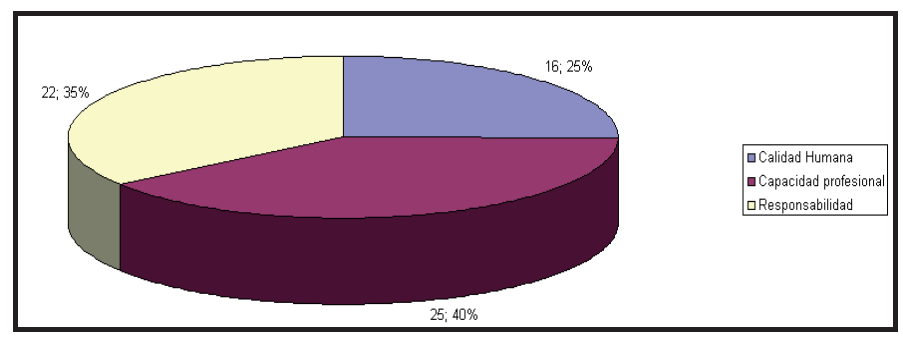

Fuente: Grupo investigador

Los aspectos que destacan los empresarios habla muy bien de la formación integral de los egresados: capacidad profesional, responsabilidad y calidad humana son calificativos muy dicientes de la satisfacción que ellos expresan respecto de la calidad personal y profesional de los contadores públicos de la Universidad Santo Tomás, seccional Bucaramanga.

- Participación del contador público que labora en la empresa, en actividades complementarias que reflejan la interdisciplinariedad y la importancia del trabajo en equipo en el que deben participar los profesionales.

Tabla 5. Participación del contador público en la actividad empresarial

\begin{tabular}{|c|c|c|}
\hline Planes de mejoramiento de procesos administrativos & 8 & $61 \%$ \\
\hline Manuales de procedimiento & 3 & $19 \%$ \\
\hline ISO 9000 & 2 & $12 \%$ \\
\hline Organización y mejoramiento continuo de cada miembro de la empresa & 1 & $6 \%$ \\
\hline Contabilidad Sistematizada & 1 & $6 \%$ \\
\hline Replanteamiento del Dpto. Financiero & 1 & $6 \%$ \\
\hline Total & 16 & $100 \%$ \\
\hline
\end{tabular}

Fuente: Grupo investigador 
Se puede observar la participación de un importante porcentaje de los egresados en procesos administrativos diferentes de los relacionados fundamentalmente con la parte técnica contable, quienes han participado en estos procesos el aspecto más frecuente es el proceso de implementación de normas de calidad, normas ambientales y procesos de seguridad industrial.

\section{CONCLUSIONES}

La existencia y vigencia de la Universidad Santo Tomás y su Facultad de Contaduría Pública ha sido determinante para la región, su influencia e impacto en la comunidad es evidente y así lo reconocen las autoridades y los diferentes estamentos sociales, el análisis de los resultados permite confirmar esta apreciación que se respalda en los siguientes aspectos:

- La vinculación y participación de los egresados en el sector productivo representa un impacto social y humano que se materializa con el ejercicio profesional como asesores, consultores, revisores fiscales, auditores internos y externos, jefes de departamento, entre otros, que se puede evidenciar con el nivel de ocupación profesional, la satisfacción de los empresarios, las posibilidades y proyección que su formación ha representado a nivel individual y su grupo familiar.

- El sostenimiento del programa de manera ininterrumpida durante treinta y seis años; a pesar de las vicisitudes que se ha tenido que enfrentar durante su existencia como crisis económicas, incremento notable de ofertas académicas en este campo y en otras áreas, el desarrollo tecnológico que ha permitido la oferta y proliferación de otras alternativas de formación, a pesar de lo cual, siempre ha existido interés en la sociedad y demanda de servicios educativos para respaldar su permanencia y vigencia.

- El nivel de ocupación de los egresados quienes han encontrado en el sector público y/o privado la oportunidad de aplicar los conocimientos adquiridos en su formación profesional, al servicio de otras personas o en beneficio propio en el desarrollo de sus propias empresas.

- La manifestación expresa de los egresados quienes han encontrado que los fundamentos técnicos, científicos y de formación integral que ofrece la Universidad, han sido factor fundamental para el éxito de su labor y desempeño profesional, en un ambiente de competitividad frente a profesionales de otras instituciones. 
- Los sentimientos de gratitud, agradecimiento, reconocimiento, nostalgia y liderazgo expresados por los egresados hacia la institución por la formación recibida y la oportunidad que ésta ha representado en su vida para la materialización de sus expectativas y el cumplimiento de sus objetivos y metas a nivel personal y de su grupo familiar.

- La expresión de los empresarios quienes reconocen en el profesional egresado de la Facultad de Contaduría Pública la capacidad profesional, su responsabilidad y calidad humana al servicio de las empresas y del entorno laboral en el que se desempeñan.

- La estabilidad que se observa en la vinculación de los egresados con las empresas, el $84 \%$ de los empresarios expresan haber tenido vinculación con egresados por más de un año.

- El impacto social favorable que ha representado para la familia de los egresados, que se desprende de la oportunidad de trabajo, estabilidad y el grado de satisfacción personal manifestado.

- La aceptación de los egresados de los programas de formación en postgrados que se ofrecen como complemento y profundización de su formación profesional.

- El $47 \%$ de los egresados no han realizado estudios de posgrado y el nivel de estudios en maestría es muy limitado. Esta situación es comprensible dado que en la región y el país la oferta de estos programas en el área profesional es mínima, con excepción del área de finanzas o de tributación, no existen otras opciones lo cual representa para la Universidad una oportunidad para desarrollo de programas de maestría que satisfagan esta necesidad

- Los egresados son el producto final de las instituciones educativas, Ellos, a su vez, se convierten en sus principales promotores, donde quiera que desempeñen su labor profesional, son testimonio expreso de la calidad de la formación que se imparte en ellas y un referente fundamental del grado de aceptación que se tiene por parte del sector productivo y la comunidad en general.

- Entre las sugerencias de mejoramiento al proceso de formación que expresan los egresados está el relacionado con una mayor práctica empresarial, para el $20 \%$ de los encuestados este es un aspecto sobre el cual se debe dar un mayor énfasis y para el 17\% el fortalecimiento de principios éticos, son de igual manera, una necesidad para enfrentar con éxito las dificultades evidentes que el mundo actual ofrece. Para los empresarios el aspecto más destacable es el fortalecimiento de la formación es la utilización de herramientas tecnológicas, el $32 \%$ de ellos consideran que este aspecto debe fortalecerse, otro aspecto expresado por el $17 \%$ de ellos, es el mejoramiento de la comunicación verbal 
y escrita, aspecto sobre el cual los profesionales modernos, de manera general, presentan algunas debilidades.

\section{RECOMENDACIONES}

Es indudable que en un proceso de crecimiento siempre hay espacio para el mejoramiento continuo, algunos aspectos que el desarrollo del proyecto permite identificar son:

- Se considera recomendable para la Facultad de Contaduría Pública, evaluar con alguna frecuencia el impacto de sus egresados en cuanto permite afianzar las fortalezas que le otorgan en su preparación al egresado con el fin de hacerlo más competitivo en el mercado, al tiempo que realimentar el proceso y renovar los principios y propósitos planteados en la misión institucional los cuales fundamentaron la decisión de crear el programa y mantener su vigencia, justificar su permanencia a pesar de la transformación de la comunidad, el avance tecnológico, el cambio de condiciones y las nuevas circunstancias que afectan la formación académica de profesionales en todas las áreas y en particular de la contaduría pública.

- Lograr una mayor relación entre la teoría y la práctica de tal forma que el estudiante pueda enfrentar con mayores argumentos el ejercicio profesional. Fundamentar la formación profesional con el apoyo de herramientas tecnológicas que posibiliten un mejor desempeño profesional, fortalecer la formación del profesional de la contaduría pública en aspectos como la expresión oral y escrita y el comportamiento social, y reforzar la formación ética profesional del contador público.

- Hacer efectiva la participación de los egresados en los órganos de dirección de la Facultad como el Consejo de Facultad y el Comité Curricular con el propósito de contar con la opinión y participación de los egresados en la orientación general de la Facultad.

- Fortalecer los procesos de investigación para crear las condiciones que permitan ofrecer maestrías relacionadas con el área profesional en el mediano plazo como una etapa para llegar al doctorado mediante la capacitación docente en la utilización de la tecnología y el desarrollo de procesos de evaluación y de investigación.

- Presentar propuestas de renovación curricular que permitan implementar e incorporar las nuevas tendencias del pensamiento, los avances tecnológicos y las nuevas metodologías, que garanticen su vigencia de acuerdo con las exigencias de la sociedad, los convenios y las tendencias internacionales. 
- Proyectar la internacionalización del programa y su proyección allende las fronteras para fortalecer los procesos académicos en general procurando el intercambio y la movilidad de la comunidad universitaria.

\section{Referencias}

Bedoya, José Iván. Pedagogía. ¿Enseñar a pensar? Reflexión filosófica sobre el proceso de enseñar. Bogotá: ECOE Ediciones, 1998

Cardona Artehaga, John y ZAPATA, Miguel Ángel. Educación Contable, actualidad y prospectiva. Medellín: Universidad de Antioquia, 2005

Contreras Hernández, Mauricio. Estándares educativos: propuesta de apropiación y adecuación. Bogotá: ABC del Educador, 2004

Cruz Tomé, M. A. (2000). De la formación pedagógica inicial y permanente del profesor universitario en España: reflexiones y propuesta. En: Revista Interuniversitaria de Formación del Profesorado. No. 38 (1999); p.19-35

Gallego Badillo, Rómulo. Saber pedagógico: una visión alternativa. Bogotá Mesa Redonda

Instituto Colombiano de Normas Técnicas. Normas colombianas para la presentación de trabajos de investigación. Quinta actualización. Santafé de Bogotá D.C. : ICONTEC, 2002. 126 p. NTC 1486

Lerna, Héctor Daniel. Presentación de Informes: el documento final de Investigación. Bogotá: ECOE Ediciones, 2003

Lozada Ortiz, Álvaro y Moreno M., Heladio. Ciencias básicas aplicadas al aula. Bogotá: ABC del Educador, 2002

Resolución 3459 del 30 de Diciembre de 2.003. Definición de las características específicas de calidad para los Programas de formación profesional de Pregrado en Contaduría Pública

Tobon, Sergio. Formación basada en competencias. Portafolio de Competencias. Medellín E. Book 2.003

Universidad Externado de Colombia.@puntes contables. El Contador como ser humano; campo profesional. Vol. 6. Facultad de Contaduría Pública. 2004

Universidad Externado de Colombia. Sección de Competencias. Vol. 5. Facultad de Contaduría Pública, 2004

Universidad Santo Tomás. Estructura Operativa del Sistema de Investigación en la Universidad Santo Tomás, Seccional Bucaramanga 


\section{PAUTAS PARA COLABORADORES}

La Revista Lebret, de la División de Ciencias Económicas, Administrativas y Contables de la Universidad Santo Tomás considera la publicación de trabajos inéditos que aporten a las áreas de las ciencias sociales, en particular la economía, la administración y la contaduría. Estos textos deben ser producto de investigaciones realizadas. La revista acoge los criterios de Colciencias-Publindex para artículos científicos:

1) Proyectos finalizados de investigación científica (Artículos de investigación): documento que presenta los resultados originales de proyectos de investigación y la estructura utilizada contiene: introducción, metodología, resultados y conclusiones.

2 Reflexiones analíticas sobre un problema particular, con base en fuentes originales (Artículos de reflexión): documento que presenta resultados de investigación finalizada, desde una perspectiva analítica, interpretativa del autor, sobre un tema específico.

3) Revisiones bibliográficas (Artículo de revisión): documento de investigación terminada donde se analizan los resultados de investigaciones publicadas o sin publicar, sobre un campo específico, para dar cuenta de las tendencias y avances del mismo mediante por lo menos cincuenta referencias.

La revista incluirá también reseñas de libros en español o traducciones que no hayan sido publicados en otras revistas.

El autor debe adjuntar un resumen de la hoja de vida que señale sus títulos académicos, cargos desempeñados, publicaciones recientes y correo electrónico. El artículo y los archivos adjuntos se reciben en la siguiente dirección: revistalebret@gmail.com. La recepción de artículos se realiza desde el 30 de abril y hasta el 30 mayo de cada año.

\section{Pautas para artículos:}

1. Las contribuciones deben se inéditas, presentadas en formato Word, cuadros y gráficos en Excel, versión office 2003, con claridad en las convenciones. Los archivos deben señalar el lugar y página donde se insertan los cuadros y gráficos o especificar los cuadros anexos. Las imágenes o mapas deben tener una resolución de por lo menos 300 dpi y estar en formato .tif o .jpg

2. Los trabajos tendrán una extensión entre 5.000 y 10.000 palabras, tamaño carta, incluidas notas y referencias bibliográficas; la letra Times New Roman 12 puntos, interlineado doble espacio y márgenes izquierdo de $4 \mathrm{~cm}$ y $2,5 \mathrm{~cm}$ en la parte superior, inferior derecha de cada página. 
3. Las reseñas de libros publicados no pueden exceder las 1.200 palabras.

4. La extensión del resumen en español e inglés debe tener entre 6 y 10 líneas. El autor especificará tres o cuatro palabras claves.

5. Los artículos deben ser originales, con redacción clara, precisa e impersonal.

6. El título del artículo debe ser preciso y exponer el tema del trabajo.

7. El resumen del artículo debe estar redactado en tercera persona y en tiempo presente. Contiene: la pregunta, el marco teórico, la metodología, los principales hallazgos y las conclusiones.

8. Las notas de pie de página se emplearán para hacer aclaraciones o explicitar datos adicionales, deben aparecer numeradas y ser lo má breve posible.

9. Las referencias bibliográficas (citas) se realizarán en el cuerpo del texto entre paréntesis. Por ejemplo (apellido, año) en citas textuales se agrega la página (apellido, año, p.) Ejemplo: (González, 2008, 65).

10. En las citas textuales menores de 40 palabras, el párrafo citado irá entre comillas, en el cuerpo del texto. Las citas textuales con más de 40 palabras, comienzan en un nuevo renglón con una sangría de 5 espacios en el margen izquierdo y sin comillas.

11. Para citar un estudio elaborado por más de 2 personas y menos de 6 debe escribirse el apellido de todos los autores. Si la cita va al inicio del párrafo, por ejemplo: Therán, González, Daza y Botache (2004) encontraron que los factores de rendimiento de las frutas dependen de la calidad del suelo. Al final del párrafo por ejemplo: los factores de rendimiento de las frutas dependen de la calidad del suelo (Therán, González, Lozano \& Botache, 2004). En adelante, cada vez que se mencione el texto de estos autores se escribirá el apellido del primer autor, seguido por la palabra et al. y la fecha.

12. Las citas de periódicos deben ser incorporadas dentro del texto entre paréntesis así: Titulo de la fuente, día mes año, página.

13. Las referencias bibliográficas completas van al final del texto, por orden alfabético según el apellido del autor. Se denomina referencias bibliográficas, en cambio de bibliografía, estas deben corresponder con cada cita.

14. Libros: apellidos e iniciales del nombre de los autores; año de la edición utilizada, título del libro en cursiva, ciudad de la publicación, país: casa editora, (año de la publicación original, si la obra ha tenido varias ediciones). Ejemplo: Therán, C. (1997). La productividad en la manufactura, Bogotá, Colombia: Fondo de Cultura Económica. Si la obra ha tenido varias ediciones: Carmen Therán, 1997, la productividad en la manufactura, Bogotá: Fondo de Cultura Económica, 2000. 
15. Artículos: apellidos e iniciales de los nombres de los autores, año de la publicación, título del artículo, nombre de la revista y volumen (si tiene) en cursiva, número, páginas del artículo. Ejemplo: Botache, R. (2001). Mercado de tierras. Cife, 56, 3136.

16. Capítulos de libro: Autor, (año), título del capítulo, en nombre de editores, nombre de libro en cursiva, páginas del capítulo, ciudad, país: editorial. Ejemplo: Gómez, E., Linares, H. (2001). Técnicas de contabilidad y negocios internacionales. En J. Santos \& Albarracín. M(Eds.). Finanzas internacionales (pp. 409-500). Bucaramanga, Colombia: Tres Culturas.

17. Los trabajos sin autor: Se incluyen solamente si es importante para el texto y se escribe entre comillas el nombre del libro; por ejemplo: ("Constitución Política de Colombia", 1991) es una referencia usada para citar decretos y leyes.

18. Las citas de un autor que cita a otro autor debe corresponder en referencias con el texto de quien hace la cita. En el cuerpo del texto se cita el autor, paréntesis, año, citados en apellido, año. Por ejemplo: al comienzo del texto: Rojas y Linares (1996 citado en Prada, González \& Botache, 2000). Al final del texto (Rojas y Linares, 1996 citado en Lozano, González \& Botache, 2000).

19. Documentos en sitio Web de una organización: Apellido, inicial de nombre. (año), titulo del texto en cursiva. Recuperado el día, mes año, del sitio Web (nombre del sitio Web): dirección virtual. Ejemplo: Caviedes, E. (2001). La administración actual. Recuperado el 13 de agosto de 2008, del sitio Web de la Asociación Colombiana de Administración: http://www.acad.or/detalle/06/id.doc1-colombia-pdf.

20 Proceso de selección de contribuciones:

21. Los documentos se recibirán mediante convocatoria abierta a la comunidad académica nacional, regional e institucional, estableciendo un tiempo límite para su recepción. Los artículos seleccionados por el Comité Editorial, serán sometidos a la evaluación por expertos, quienes dispondrán de un mes para emitir su concepto.

22. La revista dispone de una base de datos de pares internos y externos a la institución para la evaluación de cada uno de los artículos. El proceso de evaluación es confidencial, pero los comentarios de los artículos se remitirán a los autores, con las sugerencias pertinentes del Comité Editorial de la revista, en caso de requerir ajustes.

23. La revista establecerá un seguimiento editorial, con la fecha de recepción y aprobación de cada uno de los artículos; el diligenciamiento de formato de evaluación (calidad, originalidad e importancia del texto) por cada uno de los evaluadores, quienes emitirán su concepto a través de una calificación y valoración del texto. La decisión de publicación de artículos corresponde al Comité Editorial. 
Esta obra se terminó

de imprimir en Diciembre de 2009

en los talleres de Distrigraf impresores de Bucaramanga 


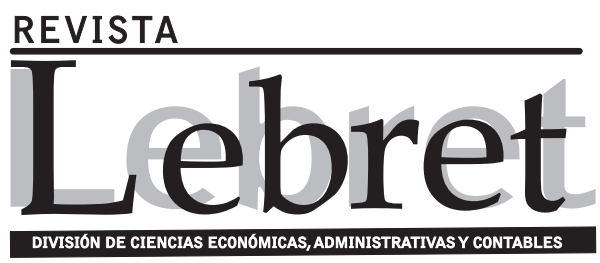

\section{CUPON DE SUSCRIPCIÓN}

\begin{tabular}{|l|}
\hline \multicolumn{1}{|c|}{ SUSCRIPTORES } \\
\hline Nombres \\
\hline Apellidos \\
\hline
\end{tabular}

\begin{tabular}{|c|c|c|c|}
\hline \multicolumn{4}{|c|}{ RESIDENCIA } \\
\hline \multicolumn{4}{|c|}{ Dirección } \\
\hline Ciudad & & País & \\
\hline Telefono & fax & & Celular \\
\hline
\end{tabular}

\section{SUSCRIPCIÓN ANUAL}

Colombia $\$ 10.000 \quad$ Exterior: US $\$ 5$

\section{SUSCRIPCIÓN A PARTIR DEL NÚMERO}

\section{ESTIMADO SUSCRIPTOR}

- Consigne en la cuenta de ahorrros número 184-91-706-0 del Banco de Bogotá a nombre de Universidad Santo Tomás

- Envie copia del recibo de consignación, junto con este cupon a la División de Ciencias Económicas y Contables de la Universidad Santo Tomás en la carrera 18 No9-27 Bucaramanga o copia a revistalebret@gmail.com 
